\title{
Produção de carvão ativado a partir de Eucalyptus dunnii para adsorção de corante azul de metileno
}

\author{
Rayssa de Medeiros Morais ${ }^{1 \star}$, Gregório Mateus Santana ${ }^{1}$, Roberto Carlos Costa Lelis ${ }^{1}$, Maria \\ Vanessa Egger Schueler ${ }^{1}$, Fernanda Lago Morbeck ${ }^{1}$, Juarez Benigno Paes ${ }^{2}$
}

${ }^{1}$ Universidade Federal Rural do Rio de Janeiro, Seropédica, Rio de Janeiro, Brasil.

${ }^{2}$ Universidade Federal do Espirito Santo, Jerônimo Monteiro, Espírito Santo, Brasil.

\begin{abstract}
RESUMO O carvão ativado é um adsorvente produzido através do processo de ativação física ou química, dando origem a um material com alta porosidade e grande área superficial, fundamental para remoção de contaminantes do meio ambiente. Diante disso, objetivou-se produzir carvão ativado a partir de Eucalyptus dunnii e aplicá-lo para adsorção do corante azul de metileno em meio aquoso. Destarte, o carvão ativado foi preparado mediante ativação física, utilizando como agente ativante o vapor d'água. Em seguida, realizadas caracterizações referentes ao rendimento gravimétrico em carvão ativado ( $\left.\mathrm{RG}_{\mathrm{CA}}\right)$, análise de área superficial $\left(\mathrm{S}_{\mathrm{BET}}\right)$, volume e diâmetro dos poros e microscopia eletrônica de varredura. Para avaliação da capacidade adsortiva foram realizados estudos de cinética e isotermas de adsorção, em diferentes concentrações do corante, e aplicados os modelos de isotermas de Langmuir e Freundlich. O material produzido apresentou $\mathrm{RG}_{\mathrm{CA}}$ de $15 \%$, $\mathrm{S}_{\mathrm{BET}}$ igual a $532,32 \mathrm{~m}^{2} \mathrm{~g}^{-1} \mathrm{e} \mathrm{morfologia}$ superficial porosa. Além disso, apresentou uma capacidade máxima de adsorção $\left(\mathrm{q}_{\mathrm{m}}\right)$ para o corante azul de metileno igual a 293,30 $\mathrm{mg} \mathrm{g}^{-1}$, se ajustando melhor ao modelo de Langmuir (monocamada). Dessa maneira, conclui-se que o material produzido obteve excelentes propriedades (texturais e adsortivas), sendo eficiente para adsorção do corante azul de metileno.

Palavras-chave: Ativação física; eucalipto; remoção de contaminantes.
\end{abstract}

\section{Production of activated carbon from Eucalyptus dunnii for adsorption of methylene blue dye}

\begin{abstract}
Activated carbon is an adsorbent produced through the process of physical or chemical activation, giving rise to a material with high porosity and large surface area, fundamental for removing contaminants in the environment. Accordingly, this study aimed to produce activated carbon from Eucalyptus dunnii and apply this activated carbon to adsorption of methylene blue dye in aqueous medium. Thus, activated carbon was prepared by physical activation, using water vapor as the activating agent. Then, we realized characterizations related to the gravimetric yield in activated carbon $\left(\mathrm{GY}_{\mathrm{AC}}\right)$, surface area analysis $\left(\mathrm{S}_{\mathrm{BET}}\right)$, pore volume and diameter and scanning electron microscopy. The adsorption capacity was evaluated from kinetic studies and adsorption isotherms at different concentrations of dye and Langmuir and Freundlich isotherm models were applied. The material produced had $15 \% \mathrm{GY}_{\mathrm{AC}}, \mathrm{S}_{\mathrm{BET}}$ equal to $532.32 \mathrm{~m}^{2} \cdot \mathrm{g}^{-1}$ and porous surface morphology. In addition, the material presented a maximum adsorption capacity $\left(\mathrm{q}_{\mathrm{m}}\right)$ for the methylene blue dye equal to $293.30 \mathrm{mg}$. $\mathrm{g}^{-1}$, adjusting better to the Langmuir (monolayer) model. Therefore, we concluded that the material produced excellent properties (textural and adsorptive), being efficient for adsorption of methylene blue dye.
\end{abstract}

Keywords: Physical activation; eucalyptus; removal of contaminants. 


\section{Introdução}

A área total de árvores plantadas no Brasil em 2016 foi de 7,84 milhões de hectares, apresentando um crescimento de 0,5\% em relação a 2015. Deste total, 5,7 milhões de hectares correspondem exclusivamente as espécies de eucaliptos, que representam mais de 70\% das áreas plantadas, localizadas em Minas Gerais com 24\%, em São Paulo com 17\% e no Mato Grosso do Sul com 15\% (IBÁ, 2017).

Dentre as inúmeras espécies plantadas, encontra-se o Eucalyptus dunnii, onde o Brasil ocupa o primeiro lugar em áreas de plantios com esta espécie, de clima temperado, com potencial de produção de mais de $50 \mathrm{~m}^{3} /$ ha.ano (FILHO et al., 2006). Em vista disso, indiscutivelmente, o Brasil aparece em destaque no panorama mundial quanto à produtividade de plantios de eucaliptos, em virtude das condições ambientais favoráveis ao desenvolvimento da cultura e ao incentivo de pesquisas destinadas para melhorias de materiais genéticos, obtidas de suas espécies e híbridos (CASTRO et al., 2016). Contudo, todo conhecimento tem se concentrado sobretudo na área de celulose e papel, mesmo que muitos outros usos podem ser dados, como por exemplo o uso da madeira de Eucalyptus dunnii para produção de carvão ativado.

O carvão ativado trata-se de um material com formação microcristalina, não grafítica, sendo um sólido amorfo, encontrado essencialmente na sua forma de pó ou granulado, que é ativado para desenvolver sua porosidade, aumentando o volume de poros e a área de superfície (GONÇALVES et al., 2006; MARSH; REINOSO, 2006; COUTO et al., 2012; NASCIMENTO et al., 2014; DANISH; AHMAD, 2018). Devido suas propriedades adsortivas tem sido amplamente utilizado em uma serie de aplicações, tais como na remoção de poluentes (NOROUZI et al., 2018; FRÖHLICH et al., 2018), usos médicos (ZAWADZKI et al., 2017; OLLIER et al., 2017), como catalisadores e para o armazenamento de gás (KOONAPHAPDEELERT et al., 2018).
Para a produção do carvão ativado são empregados usualmente dois processos: a carbonização e a ativação propriamente dita. Esses dois processos podem ser realizados separadamente ou em uma única etapa (SOUZA et al., 2016). Para a ativação, esta pode ser dividida em física ou química. $\mathrm{Na}$ ativação física, o material inicialmente carbonizado é submetido a uma gaseificação parcial e controlada a alta temperatura, utilizando agentes oxidantes na temperatura final, tais como o $\mathrm{CO}_{2}$, o vapor d'água, ou até mesmo a mistura de ambos (GONZÁLEZ-GARCÍA, 2018). Já na ativação química ocorre uma impregnação inicial no material precursor de um agente ativante químico desidratante $\left(\mathrm{H}_{3} \mathrm{PO}_{4}, \mathrm{H}_{2} \mathrm{SO}_{4}, \mathrm{ZnCl}_{2}, \mathrm{KOH}\right.$ ou $\left.\mathrm{NaOH}\right)$, sendo após isso efetivada a ativação em temperaturas menores (DANISH; AHMAD, 2018).

A maioria dos materiais carbonáceos podem ser ativados. No entanto, existe uma demanda crescente por carvões ativados produzidos a partir de materiais renováveis, bem como de subprodutos agrícolas e florestais e resíduos de forma geral (RAMBABU et al., 2015; CORREA et al., 2017; GONZÁLEZ-GARCÍA, 2018). Isto posto, via de regra, após a ativação se obtém um material com alto teor de carbono, elevada área superficial e volume de poros, sendo ainda a qualidade do material obtido alterada consoante o processo de ativação utilizado (DANISH; AHMAD, 2018).

Presentemente, os problemas de poluição ambiental têm se tornado cada vez mais alarmantes devido sobretudo à contaminação doméstica e industrial excessiva, que prejudica o meio ambiente, principalmente a água. Para solucionar esta questão, vários processos são usados para tratar a água contaminada, incluindo a tecnologia de adsorção por carvão ativado (OUHAMMOU et al., 2017). A contaminação da água ocorre quando existe o acúmulo de substâncias nocivas, como é o caso de corantes (BULUT; AYDIN, 2006), que podem ser bastantes prejudiciais à saúde humana, por 
possuírem uma estrutura molecular complexa e estável, com toxidade variável, reunindo propriedades cancerígenas, mutagênicas e alergênicas (ISLAM et al., 2017).

Assim, diante deste contexto, objetivou-se produzir e avaliar a qualidade do carvão ativado preparado fisicamente a partir da madeira de Eucalyptus dunnii, analisar suas propriedades e capacidade de adsorção para o corante azul de metileno.

\section{Material e Métodos}

\section{Produção do carvão ativado}

Foi utilizado como material precursor a madeira de Eucalyptus dunnii, fornecida pela empresa Terra Pinnus, localizada em Lages, Santa Catarina, proveniente de plantios com sete anos de idade. Para a produção do carvão ativado foi empregado o processo de ativação física direta, onde a madeira em forma de lascas foi colocada diretamente in natura no forno de ativação, eliminando a etapa de carbonização, comumente empregada, sendo ainda utilizado como agente ativante o vapor d'água.

Para ativação foi utilizado um forno rotativo da CHINO, marca Takabayashi Rica, modelo RS-S, Japão. Para o processo, empregou-se 100 gramas de material precursor que foram inseridos no reator tubular de leito fixo, com dimensões de $93 \mathrm{~mm}$ de diâmetro e $900 \mathrm{~mm}$ de comprimento. Ao forno estão acoplados uma caldeira elétrica para a obtenção do vapor d'água e um recipiente dewar para nitrogênio $\left(\mathrm{N}_{2}\right)$.

No processo foi empregada uma taxa de aquecimento de $10{ }^{\circ} \mathrm{C} \mathrm{min}^{-1}$ até temperatura final de $800^{\circ} \mathrm{C}$ e tempo de residência na temperatura final de 30 minutos (sob atmosfera de $\mathrm{N}_{2}$ com fluxo controlado de $80 \mathrm{~mL} \mathrm{~min}^{-1}$ ).
Atingida a temperatura final foi introduzido o vapor d'água com fluxo de $100 \mathrm{~mL} \mathrm{~min}{ }^{-1}$, conforme a Figura 1. O carvão ativado produzido a partir de Eucalyptus dunnii foi denominado CAE.

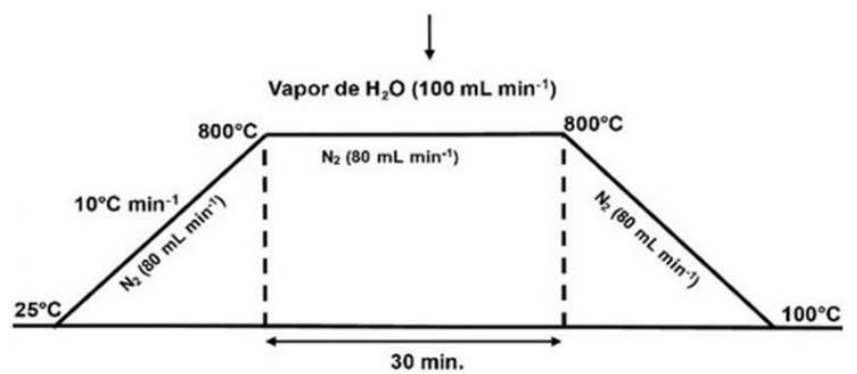

Figura 1. Rampa de aquecimento usada para produção do CAE.

Figure 1. Heating ramp used for EAC production.

O rendimento gravimétrico em carvão ativado $\left(R G_{C A}\right)$ foi calculado de acordo com a Equação 1.

$$
\mathrm{RG}_{\mathrm{CA}}(\%)=\frac{\mathrm{m}_{\mathrm{f}}}{\mathrm{m}_{0}} \times 100
$$

Em que: $\mathrm{m}_{\mathrm{f}}=$ massa seca final do carvão ativado $(\mathrm{g}) ; \mathrm{m}_{0}=$ massa seca inicial do material precursor utilizado $(\mathrm{g})$.

\section{Área superficial, volume e diâmetro dos poros}

Mediante a análise BET é possível obter informações fundamentais sobre a área superficial e estrutura porosa do carvão ativado (NASCIMENTO et al., 2014).

A caracterização textural do CAE foi realizada com os dados de adsorção em etapa gasosa obtidos com o uso de um Microporosímetro modelo ASAP 2020 (Micromeritics Instrument Corporation, Estados Unidos) conectado a um microcomputador, cujo a amostra havia sido desgaseificada, a vácuo, a $300^{\circ} \mathrm{C}$.

O método adotado emprega a técnica de adsorção de $\mathrm{N}_{2}$ a $77 \mathrm{~K}$ (para obtenção da área superficial, volume e diâmetro de poros) fundamentado nos trabalhos de BET - Brunauer, Emmett e Teller (SKAAR, 1988), representado pela Equação 2. 


$$
V_{a}=\frac{V_{m} \times C \times P}{\left(P_{0}-P\right)\left[1+(C-1)\left(\frac{P}{P_{0}}\right)\right]}
$$

Em que: $\mathrm{V}_{\mathrm{a}}=$ quantidade de gás adsorvido na pressão $\mathrm{P}(\mathrm{mL})$; $\mathrm{V}_{\mathrm{m}}=$ quantidade de gás adsorvido quando toda a superfície está coberta por uma camada monomolecular $(\mathrm{mL}) ; \mathrm{C}=$ constante relacionada à energia de interação com a superfície; e $\mathrm{P}_{0}=$ pressão de saturação do gás.

\section{Microscopia eletrônica de varredura}

As obtenções das micrografias do carvão ativado produzido foram obtidas mediante análise de Microscopia Eletrônica de Varredura (MEV), realizada em aparelho FEI Quanta 450, ao empregar tensão de $20 \mathrm{kv}$. Para isso, as amostras foram previamente montadas sobre plataforma de alumínio, com o uso de fita de carbono dupla com uma fina camada de ouro, depositada por um metalizador Emitech k550X.

\section{Aplicação para adsorção do corante azul de}

\section{metileno}

Estudos de adsorção foram realizados com a finalidade de se avaliar a aplicação do CAE produzido para adsorção do corante azul de metileno presente em meio aquoso. $\mathrm{O}$ azul de metileno tem sido comumente empregado como composto modelo para testes de adsorção, apresentando massa molar de $319,8 \mathrm{~g} \mathrm{~mol}^{-1}$ e comprimento de onda $665 \mathrm{~nm}$. Sua estrutura química é demostrada na Figura 2.

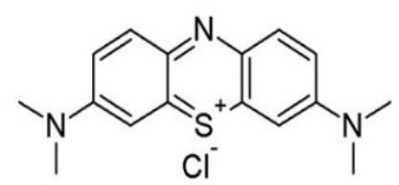

Figura 2. Estrutura química do azul de metileno Figure 2. Chemical structure of methylene blue.

\section{Cinética de adsorção}

Estudos de cinética de adsorção foram realizados em temperatura ambiente $\left(25^{\circ} \mathrm{C}\right)$, na qual foram utilizados $10 \mathrm{mg}$ de carvão ativado e $10 \mathrm{~mL}$ de solução com o corante azul de metileno na concentração inicial $\left(\mathrm{C}_{0}\right)$ de $50 \mathrm{mg} \mathrm{L}^{-1}$. Em intervalos predeterminados de 1, 2, 3, 6, 12 e 24 horas, alíquota da solução, com aproximadamente $3,5 \mathrm{~mL}$, foram retiradas e suas concentrações determinadas.

Para a determinação da concentração de equilíbrio do corante foi utilizado um aparelho espectrofotômetro, modelo UV-mini 1240, da SHIMADZU, com o comprimento de onda característico para o azul de metileno.

\section{Isoterma de adsorção}

A isoterma de adsorção foi obtida ao ser empregado 10 mg de carvão ativado e $10 \mathrm{~mL}$ da solução com diferentes concentrações do corante azul de metileno, pela qual foram inseridas em recipientes de $20 \mathrm{~mL}$ e mantidos sob agitação de $100 \mathrm{rpm}$ em uma incubadora Shaker (modelo SL 221, da SOLAB) por 24 horas, à temperatura ambiente. A concentração de equilíbrio foi obtida no espectrofotômetro, com o comprimento de onda do corante, em análise pontual de absorbância.

Foram preparadas curvas de calibração com soluções de concentrações de $25,50,100,250,500$ e $1000 \mathrm{mg} \mathrm{L}^{-1}$ para o azul de metileno. A quantidade de adsorvato adsorvido, $\mathrm{q}_{\mathrm{eq}}$, por g de adsorvente, foi calculada conforme a Equação 3.

$$
q_{e q}=\frac{\left(C_{0}-C_{e q}\right) * V}{m}
$$

Em que: $\mathrm{C}_{0}=$ concentração inicial do corante azul de metileno $\left(\mathrm{mg} \mathrm{L}^{-1}\right) ; \mathrm{C}_{\mathrm{eq}}=$ concentração do azul de metileno após atingir o equilíbrio de adsorção $\left(\mathrm{mg} \mathrm{L}^{-1}\right) ; \mathrm{V}=$ volume de adsorvato $(\mathrm{L})$; e $\mathrm{m}=$ massa de adsorvente $(\mathrm{g})$.

Os dados obtidos da isoterma foram ajustados de acordo com os modelos de isoterma de Langmuir e de Freundlich. A 
Equação 4 descreve o comportamento da isoterma de Langmuir, cuja forma linear consta na Equação 5.

$$
\begin{aligned}
& q_{e q}=\frac{q_{m} * K_{L} * C_{e q}}{1+K_{L}+C_{e q}} \\
& \frac{C_{e q}}{q_{e q}}=\frac{1}{q_{m} * K_{L}}+\frac{1}{q_{m}} * C_{e q}
\end{aligned}
$$

Em que: $\mathrm{q}_{\mathrm{eq}}=$ a quantidade adsorvida $\left(\mathrm{mg} \mathrm{g}^{-1}\right)$ para uma dada concentração do corante; $\mathrm{C}_{\mathrm{eq}}=$ concentração do corante após ter atingido o equilíbrio $\left(\mathrm{mg} \mathrm{L}^{-1}\right) ; \mathrm{q}_{\mathrm{m}}=$ a capacidade máxima de adsorção $\left(\mathrm{mg} \mathrm{g}^{-1}\right)$; e $\mathrm{K}_{\mathrm{L}}=$ constante de Langmuir.

Já a Equação 6 representa o modelo de Freundlich, a qual é convenientemente linearizada pela aplicação de logaritmo nos dois termos, conforme a Equação 7.

$$
\begin{aligned}
& q_{e q}=K_{F} * C_{e q}^{1 / n} \\
& \log q_{e q}=\log K_{F}+\frac{1}{n} \log C_{e q}
\end{aligned}
$$

Em que: $\mathrm{q}_{\mathrm{eq}}=$ a quantidade adsorvida $\left(\mathrm{mg} \mathrm{g}^{-1}\right)$ para uma dada concentração do corante; $\mathrm{C}_{\mathrm{eq}}=$ concentração do corante após ter atingido o equilíbrio $\left(\mathrm{mg} \mathrm{L}^{-1}\right) ; \mathrm{K}_{\mathrm{F}}=$ coeficiente de sorção; e $1 / \mathrm{n}=$ medida da intensidade de sorção.

\section{Resultados e Discussão}

\section{Rendimento gravimétrico em carvão ativado}

O $\mathrm{RG}_{\mathrm{CA}}$ obtido pelo CAE foi de $15 \%$, intermediário aos valores obtidos por outros carvões ativados fisicamente da literatura, a partir de subprodutos agrícolas, florestais e resíduos de forma geral, como exemplo desde espigas de milho com 8,7\% (EL-HENDAWY et al., 2001) a fibras de piaçava com 23,3\% (AVELAR et al., 2010).

Segundo Zhang et al. (2014), na ativação física ocorre o desenvolvimento da porosidade à medida que a temperatura é aumentada, sendo que acima de $650^{\circ} \mathrm{C}$, a área superficial e o volume de poros aumentam substancialmente, pois reações endotérmicas entre material / agente oxidante são favorecidas sob temperaturas altas.
Por conseguinte, quanto maiores as temperaturas empregadas $\left(800{ }^{\circ} \mathrm{C}\right.$ para o $\left.\mathrm{CAE}\right)$, menores serão os rendimentos obtidos, tendo em vista que são estes materiais lignocelulósicos, compostos essencialmente por celulose e lignina (MCKENDRY, 2002). Além disso, Rezma et al. (2017) relata que os valores menores obtidos de $\mathrm{RG}_{\mathrm{CA}}$ é um indicativo do desenvolvimento da porosidade, sobretudo da microporosidade, que aumenta o volume total de poros e também a área superficial.

\section{Área superficial, volume e diâmetro dos poros}

A análise da Figura 3 indica que o CAE produzido, ativado fisicamente com vapor d'água, apresentou uma elevada capacidade de adsorção de $\mathrm{N}_{2}$ em baixas pressões relativas $\left(\mathrm{P} / \mathrm{P}_{0}\right)$, indicando que a natureza do mesmo é microporosa. Também, verifica-se pelas isotermas de adsorção/dessorção que o CAE possui uma pequena região de histerese, que revela a presença de mesoporos em sua estrutura.

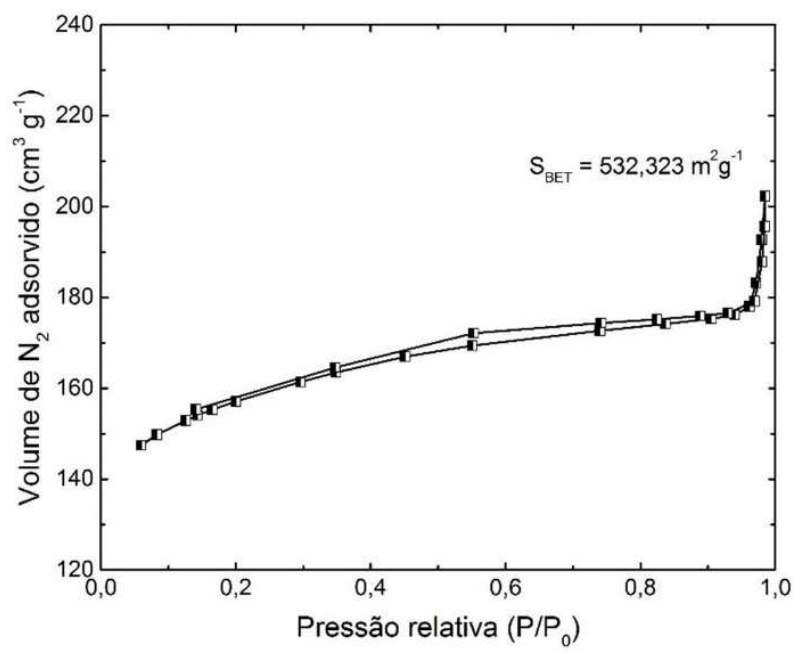

Figura 3. Isotermas de adsorção/dessorção de nitrogênio, a $77 \mathrm{~K}$, para o CAE.

Figure 3. Nitrogen adsorption / desorption isotherms, at 77 $\mathrm{K}$, for $\mathrm{EAC}$.

Na Tabela 1 estão apresentadas a área superficial BET $\left(\mathrm{S}_{\mathrm{BET}}\right)$ obtida pelo CAE produzido e de outros adsorventes utilizados para remoção de contaminantes. 
Observa-se que o CAE apresentou uma área superficial relativamente alta (nota-se que foi aplicado um tempo de residência apenas de 30 minutos) quando comparado aos valores de $\mathrm{S}_{\mathrm{BET}}$ obtidos na literatura, que varia de 364 a $565 \mathrm{~m}^{2} \mathrm{~g}^{-1}$. Destarte, de maneira geral, o CAE produzido apresentou propriedades texturais tais como área $S_{B E T}$ de $532,32 \mathrm{~m}^{2} \mathrm{~g}^{-1}$, área superficial de microporos de $408,78 \mathrm{~m}^{2} \mathrm{~g}^{-1}$ e área superficial externa de $123,54 \mathrm{~m}^{2} \mathrm{~g}^{-1}$. O volume total de poros obtidos foi de $0,30 \mathrm{~cm}^{3} \mathrm{~g}^{-1}$; com volume de microporos de $0,18 \mathrm{~cm}^{3} \mathrm{~g}^{-1}$ e um volume de mesoporos de $0,10 \mathrm{~cm}^{3} \mathrm{~g}^{-1}$, sendo o diâmetro médio dos poros obtidos de 22,73 A.

\section{Microscopia eletrônica de varredura}

A morfologia superficial do CAE produzido foi modificada após o processo de ativação, sendo a mesma composta por poros de diferentes tamanhos e formas (Figura 4a), sendo ainda bem distribuídos por toda superfície do material (Figura 4b).

Tabela 1. Área superficial $S_{\text {BET }}$ por diferentes materiais precursores.

Table 1. $S_{\text {BET }}$ surface area by different precursor materials.

\begin{tabular}{|c|c|c|c|c|}
\hline Material precursor & Tipo de ativação & $\begin{array}{c}\text { Temperatura e tempo } \\
\text { de residência }\end{array}$ & $\begin{array}{l}\text { Área } S_{\mathrm{BET}} \\
\left(\mathbf{m}^{2} \mathbf{g}^{-1}\right)\end{array}$ & Referência \\
\hline Eucalyptus dunnii & Física com vapor d'água & $800^{\circ} \mathrm{C} / 30 \mathrm{~min}$. & 532,32 & Este trabalho \\
\hline $\begin{array}{c}\text { Resíduos de Apuleia } \\
\text { leiocarpa }\end{array}$ & Física com $\mathrm{CO}_{2}$ & $850^{\circ} \mathrm{C} / 1 \mathrm{~h}$ & 564,90 & Nobre et al. (2015) \\
\hline Pecíolo de Palmeira & Física com $\mathrm{CO}_{2}$ & $850^{\circ} \mathrm{C} / 30 \mathrm{~min}$. & 546,00 & Rezma et al. (2017) \\
\hline Casca de Nozes & Química com $\mathrm{ZnCl}_{2}$ & $600^{\circ} \mathrm{C} / 2 \mathrm{~h}$ & 542,00 & Costa et al. (2015) \\
\hline Resíduos de café & Química com $\mathrm{ZnCl}_{2}$ & $500^{\circ} \mathrm{C} / 3 \mathrm{~h}$ & 521,50 & Brum et al. (2008) \\
\hline Sabugo de milho & Química com $\mathrm{ZnCl}_{2}$ & $500{ }^{\circ} \mathrm{C} / 3 \mathrm{~h}$ & 501,00 & Sales et al. (2015) \\
\hline Candeia & Física com $\mathrm{CO}_{2}$ & $900{ }^{\circ} \mathrm{C} / 1 \mathrm{~h}$ & 364,00 & Borges et al. (2016) \\
\hline
\end{tabular}
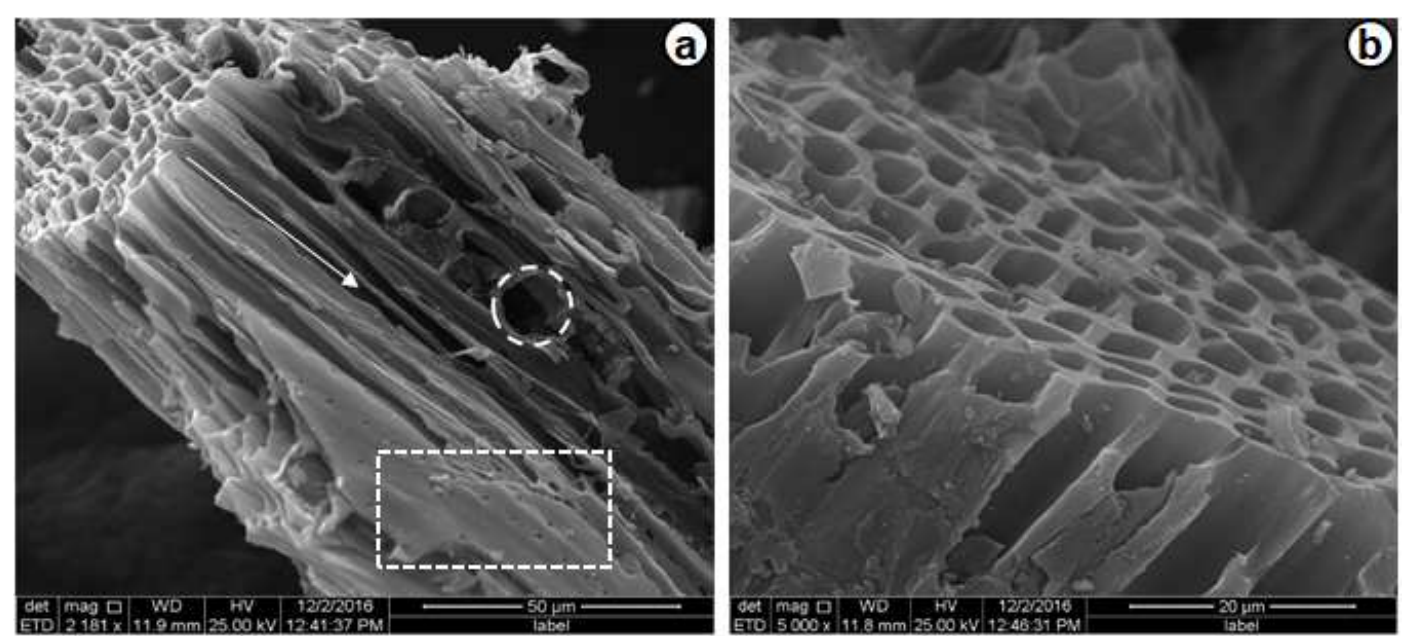

Figura 4. Morfologia superficial do CAE.

Figure 4. Surface morphology of the EAC. 
A presença de muitos poros de abertura permite a difusão rápida dos contaminantes até as regiões mais internas do CAE produzido. Ademais, o processo de ativação física direta e as variáveis do processo (temperatura final, tempo de residência e agente oxidante) promoveram uma limpeza de superfície no material.

\section{Cinética de adsorção}

A cinética de adsorção é realizada a fim de se analisar o tempo necessário para a adsorção do contaminante (SONG et al., 2013). Em vista disso, observa-se que ocorre rápida remoção do corante azul de metileno durante as primeiras horas de reação (Figura 5). Em 6 h de reação, mais de $50 \%$ do corante azul de metileno já havia sido removido e com $24 \mathrm{~h}$ o sistema já estava próximo ao equilíbrio de adsorção, com praticamente todos os sítios ativos do CAE produzido preenchidos pelo corante.

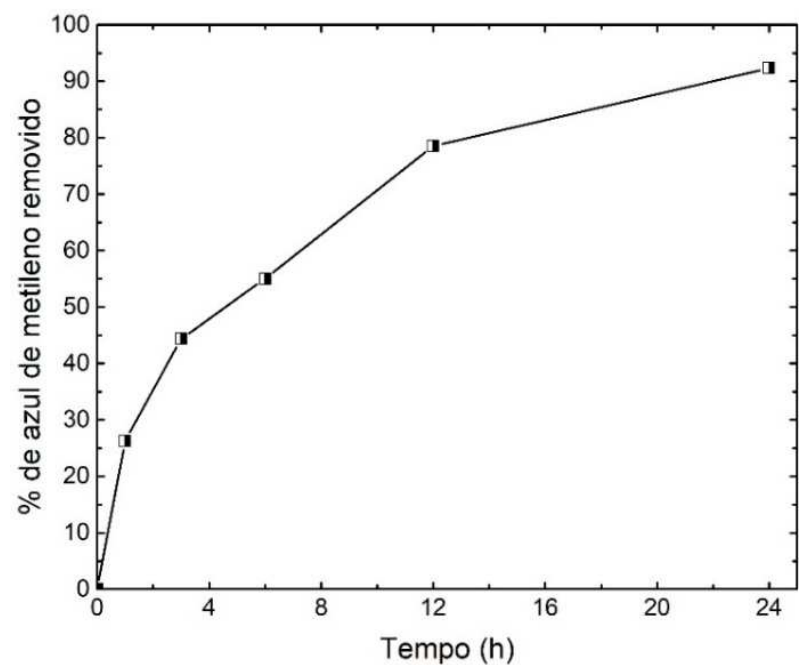

Figura 5. Cinética de adsorção para o azul de metileno para o tempo de contato adsorvato/adsorvente $(10 \mathrm{mg}$ de CA; $10 \mathrm{~mL}$ de solução $50 \mathrm{mg} \mathrm{L}^{-1} ; 25^{\circ} \mathrm{C}$ ).

Figure 5. Adsorption kinetics of methylene blue for adsorbate/adsorbent contact time $(10 \mathrm{mg} \mathrm{AC}, 10 \mathrm{~mL}$ solution $\left.50 \mathrm{mg} \mathrm{L}^{-1} ; 25^{\circ} \mathrm{C}\right)$.

\section{Isoterma de adsorção}

A isoterma de adsorção do corante azul de metileno é apresentada na Figura 6 e os parâmetros físico-químicos de adsorção obtidos a partir dos ajustes aos modelos de isotermas de Langmuir e Freundlich descritos na Tabela 2.

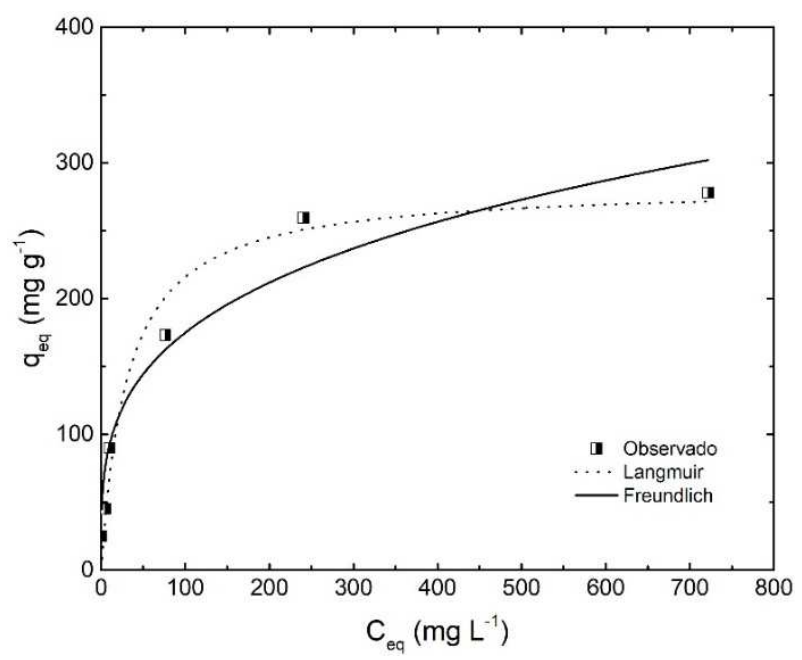

Figura 6. Isoterma de adsorção do azul de metileno.

Figure 6. Methylene blue adsorption isotherm.

Tabela 2. Parâmetros obtidos para adsorção de azul de metileno.

Table 2. Parameters obtained for methylene blue adsorption.

\begin{tabular}{cccccccc}
\hline \multirow{2}{*}{ Adsorvente } & \multicolumn{3}{c}{$\begin{array}{c}\text { Parâmetros de } \\
\text { Langmuir }\end{array}$} & \multicolumn{3}{c}{$\begin{array}{c}\text { Parâmetros de } \\
\text { Freundlich }\end{array}$} \\
\cline { 2 - 7 } & \multicolumn{2}{c}{$\mathbf{q}_{\mathbf{m}}$} & $\mathbf{K}_{\mathbf{L}}$ & $\mathbf{R}^{2}$ & $\mathbf{K}_{\mathbf{F}}$ & $\mathbf{1} / \mathbf{n}$ & $\mathbf{R}^{2}$ \\
\hline \multirow{2}{*}{ CAE } & 293,30 & 0,032 & 0,957 & 48,91 & 0,28 & 0,904
\end{tabular}

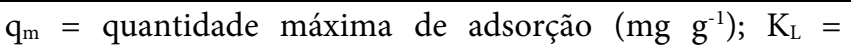
constante de Langmuir $\left(\mathrm{L} \mathrm{mg}^{-1}\right) ; \mathrm{K}_{\mathrm{F}}=$ constante de Freundlich $\left[\left(\mathrm{mg} \mathrm{L}^{-1}\right)\left(\mathrm{L} \mathrm{mg}^{-1}\right)^{1 / \mathrm{n}}\right] ; 1 / \mathrm{n}=$ parâmetro de Freundlich; e $\mathrm{R}^{2}$ = coeficiente de determinação.

Pelas isotermas e valores de $\mathrm{R}^{2}$ obtidos, nota-se que o CAE produzido possui melhor ajuste para o modelo de isoterma de Langmuir, que indica a formação de uma monocamada do corante azul de metileno sobre a superfície do CAE, na qual todos os sítios são idênticos e energicamente equivalentes (AHSAINE et al., 2018).

O CAE produzido apresentou uma eleva capacidade máxima de adsorção $\left(\mathrm{q}_{\mathrm{m}}\right)$ para o corante azul de metileno 
Tabela 3. Capacidade máxima de adsorção $\left(\mathrm{q}_{\mathrm{m}}\right)$ de $\mathrm{AM}$ por vários materiais.

Table 3. Maximum adsorption capacity ( $\mathrm{qm}$ ) of MB by several materials.

\begin{tabular}{ccccc}
\hline Material Precursor & Tipo de ativação & $\begin{array}{c}\text { Temperatura e tempo } \\
\text { de ativação }\end{array}$ & $\mathbf{q}_{\mathbf{m}}\left(\mathbf{m g} \mathbf{~ g}^{-1}\right)$ & Referência \\
\hline Eucalyptus dunnii & Física com vapor d'água & $800^{\circ} \mathrm{C} / 30 \mathrm{~min}$. & 293,30 & Este trabalho \\
Casca de buriti & Química com $\mathrm{ZnCl}_{2}$ & $700^{\circ} \mathrm{C} / 1,5 \mathrm{~h}$ & 274,62 & Pezoti et al. (2014) \\
Piaçava & Física com vapor d'água & $850^{\circ} \mathrm{C} / 1 \mathrm{~h}$ & 176,46 & Avelar et al. (2010) \\
Sabugo de milho & Química com $\mathrm{ZnCl}_{2}$ & $500^{\circ} \mathrm{C} / 3 \mathrm{~h}$ & 128,82 & Sales et al. (2015) \\
Casca de nozes & Química com $\mathrm{ZnCl}_{2}$ & $600^{\circ} \mathrm{C} / 2 \mathrm{~h}$ & 104,00 & Costa et al. (2015) \\
Eucalyptus sp. & Física com $\mathrm{CO}_{2}$ & $850^{\circ} \mathrm{C} / 1 \mathrm{~h}$ & 32,00 & Couto et al. (2012) \\
\hline
\end{tabular}

(AM) igual a $293,30 \mathrm{mg} \mathrm{\textrm {g } ^ { - 1 }}$, sendo este resultado superior aos valores de $\mathrm{q}_{\mathrm{m}}$ obtidos para carvões ativados preparados a partir de diferentes materiais precursores da literatura, que varia de 32 a $275 \mathrm{mg} \mathrm{g}^{-1}$ (Tabela 3 ).

\section{Conclusões}

De acordo com os dados experimentais, conclui-se que madeira de Eucalyptus dunnii pode ser usada de modo eficiente para produção de carvão ativado. Além disso, o CAE produzido pode ser aplicado como adsorvente para o corante azul de metileno presente em meio aquoso, tendo o mesmo apresentando excelentes propriedades texturais e adsortivas, além de possuir melhor ajuste ao modelo de Langmuir (adsorção em monocamada) e obtido uma rápida remoção do corante.

\section{Referências}

AHSAINE, H. A.; ZBAIR, M.; ANFAR, Z.; NACIRI, Y.; EL ALEM, N.; EZAHRI, M. Cationic dyes adsorption onto high surface area 'almond shell'activated carbon: Kinetics, equilibrium isotherms and surface statistical modeling. Materials Today Chemistry, v. 8, p. 121-132, 2018.

AVELAR F. F.; BIANCHI, M. L.; GONÇALVES, M.; MOTA, E. G. The use of piassava fibers (Attalea funifera) in the preparation of activated carbon. Bioresource Technology, v. 101, n. 12 , p. $4639-4645,2010$

BORGES, W. M. S.; ARANTES, A. C. C.; CASTRO, G. M. M.; BIANCHI, M. L.; NOBRE, J. R. C.; RESENDE, E. C.; CASTRO, J. P.; GUERREIRO, M. C. Produção, caracterização e avaliação da capacidade adsortiva de carvões ativado em forma de briquete. Revista Matéria, Rio de Janeiro, v. 21, n. 4, p. 930 - 942, 2016.

BRUM, S. S.; BIANCHI, M. L.; SILVA, V. D.; GONÇALVES, M.; GUERREIRO, M. C.; OLIVEIRA, L. C. A. Preparação e caracterização de carvão ativado produzido a partir de resíduos do beneficiamento do café. Quimica Nova, v. 31, n. 5, p. 1048-1052, 2008.

BULUT, Y.; AYDIN, H. A. kinetics and thermodynamics study of methylene blue adsorption on wheat shells. Desalination, v. 194, n. 1, p. 259-267, 2006.

CASTRO, C. A. O; RESENDE, R. T.; BHERING, L. L.; CRUZ, C. D. Breve histórico do melhoramento genético do eucalipto no Brasil sob a ótica dos avanços biométricos. Ciência Rural, v. 46, n. 9, p. 1585-1593, 2016.

CORREA, C. R.; OTTO, T.; KRUSE, A. Influence of the biomass components on the pore formation of activated carbon. Biomass and bioenergy, v. 97, p. 53-64, 2017.

COSTA, P. D.; FURMANSKI, L. M.; DOMINGUINI, L. Produção, Caracterização e Aplicação de Carvão Ativado de Casca de Nozes para Adsorção de Azul de Metileno. Revista Virtual de Química, v. 7, n. 4, p. 1272-1285, 2015. 
COUTO, G. M.; DESSIMONI, A. L. D. A.; BIANCHI, M. L.; PERÍGOLO, D. M.; TRUGILHO, P. F. Use of sawdust Eucalyptus sp. in the preparation of activated carbons. Ciência e Agrotecnologia, v. 36, n. 1, p. 69-77, 2012.

DANISH, M.; AHMAD, T. A review on utilization of wood biomass as a sustainable precursor for activated carbon production and application. Renewable and Sustainable Energy Reviews, v. 87, p. 1-21, 2018.

EL-HENDAWY, ABDEL-NASSER A.; SAMRA, S. E.; GIRGIS, B. S. Adsorption characteristics of activated carbons obtained from corncobs. Colloids and Surfaces A: Physicochemical and Engineering Aspects, v. 180, n. 3, p. 209-221, 2001.

FRÖHLICH, A. C.; OCAMPO-PÉREZ, R.; DIAZ-BLANCAS, V.; SALAU, N. P. G.; DOTTO, G. L. Three-dimensional mass transfer modeling of ibuprofen adsorption on activated carbon prepared by sonication. Chemical Engineering Journal, v. 341, p. 65-74, 2018.

GONÇALVES, G. C; SCOLIN MENDES, E.; CURVELO PEREIRA, N.; DE SOUSA, J. C.L. Produção de carvão ativado a partir de bagaço e melaço de cana-de-açúcar. Acta Scientiarum. Technology, v. 28, n. 1, 2006.

GONZÁLEZ-GARCÍA, P. Activated carbon from lignocellulosics precursors: A review of the synthesis methods, characterization techniques and applications. Renewable and Sustainable Energy Reviews, 2018.

FILHO, E. P.; SANTOS, P. E. T.; FERREIRA, C. A. Eucaliptos indicados para plantio no Estado do Paraná. 1.ed. Colombo-PR: Embrapa Florestas, 2006. 45p.

IBÁ - INDÚSTRIA BRASILEIRA DE ÁRVORES. Relatório IBÁ 2017. São Paulo: IBÁ, 2017.

ISLAM, M. A.; SABAR, S.; BENHOURIA, A.; KHANDAY, W. A.; ASIF, M.; HAMEED, B. H. Nanoporous activated carbon prepared from karanj (Pongamia pinnata) fruit hulls for methylene blue adsorption. Journal of the Taiwan Institute of Chemical Engineers, v. 74, p. 96-104, 2017.

KOONAPHAPDEELERT, S.; MORAN, J.; AGGARANGSI, P.; BUNKHAM, A. Low pressure biomethane gas adsorption by activated carbon. Energy for Sustainable Development, v. 43, p. 196-202, 2018.

MARSH, H.; REINOSO, F. R. Activated carbon. [s.l]: Elsevier, 2006. 536p.
MCKENDRY, P. Energy production from biomass (part 1): overview of biomass. Bioresource technology, v. 83, n. 1, p. 37-46, 2002.

NASCIMENTO, R. F.; LIMA, A. C. A.; VIDAL, C. B.; MELO, D. Q.; RAULINO, G. S. C. Adsorção: Aspectos teóricos e aplicações ambientais. Biblioteca de Ciências e Tecnologia. Fortaleza: Imprensa Universitária, 2014. 256p.

NOBRE, J. C. R.; PAZ CASTRO, J.; BIANCHI, M. L.; SILVA BORGES, W. M.; TRUGILHO, P. F.; CABRAL MOULIN, J.; NAPOLI, A. Produção de carvão ativado de resíduo madeireiro da região Amazônica. Scientia Forestalis, Piracicaba, v. 43, n. 108, p. 895-906, dez. 2015.

NOROUZI, S.; HEIDARI, M.; ALIPOUR, V.; RAHMANIAN, O.; FAZLZADEH, M.; MOHAMMADIMOGHADAM, F; NOURMORADI H.; GOUDARZI, B; DINDARLOO, K. Preparation, characterization and $\mathrm{Cr}$ (VI) adsorption evaluation of $\mathrm{NaOH}$-activated carbon produced from Date Press Cake; an agro-industrial waste. Bioresource technology, v. 258, p. 48-56, 2018.

OLLIER, E.; HODIN, S.; LANOISELÉE, J.; ESCAL, J.; ACCASSAT, S.; DE MAGALHAES, E.; BASSET, T.; BERTOLETTI, L.; MISMETTI, P. DELAVENNE, X. Effect of activated charcoal on rivaroxaban complex absorption. Clinical pharmacokinetics, v. 56, n. 7, p. 793-801, 2017.

OUHAMMOU, M.; LAHNINE, L.; MGHAZLI, S.; HIDAR, N.; BOUCHDOUG, M.; JAOUAD, A.; MANDI, L; MAHROUZ, M. Valorisation of cellulosic waste basic cactus to prepare activated carbon. Journal of the Saudi Society of Agricultural Sciences, 2017.

RAMBABU, N.; RAO, B. V. S. K.; SURISETTY, V. R.; DAS, U.; DALAI, A. K. Production, characterization, and evaluation of activated carbons from de-oiled canola meal for environmental applications. Industrial Crops and Products, v. 65, p. 572-581, 2015.

REZMA, S.; BIROT, M.; HAFIANE, A.; DELEUZE, H. Physically activated microporous carbon from a new biomass source: Date palm petioles. Comptes Rendus Chimie, v. 20, n. 9-10, p. 881-887, 2017.

SALES, P. F.; BERTOLI, A. C.; PINTO, F. M.; MAGRIOTIS, Z. M. Produção, Caracterização e Aplicação do Carvão Ativado Obtido a partir do Sabugo de Milho: A Busca pelo Reaproveitamento de um Resíduo Agroindustrial. Revista Virtual de Química, v. 7, n. 4, p. 1174-1188, 2015. 
SKAAR, C. Wood-water relations. New York: Springer (Springer Series in Wood Science), 1988.

SONG, X.; ZHANG, Y.; YAN, C.; JIANG, W.; CHANG, C. The Langmuir monolayer adsorption model of organic matter into effective pores in activated carbon. Journal of colloid and interface science, v. 389, n. 1, p. 213-219, 2013.

ZHANG, Y. J.; XING, Z. J.; DUAN, Z. K.; LI, M.; WANG, Y. Effects of steam activation on the pore structure and surface chemistry of activated carbon derived from bamboo waste. Applied Surface Science, v. 315, p. 279-286, 2014.

ZAWADZKI, A.; JOHNSON, L. B.; BOHE, M.; JOHANSSON, C.; EKELUND, M.; NIELSEN, O. H. An open prospective study evaluating efficacy and safety of a new medical device for rectal application of activated carbon in the treatment of chronic, uncomplicated perianal fistulas. International journal of colorectal disease, v. 32 , n. 4 , p. 509-512, 2017. 